\title{
ELECTROCHEMICAL AND OPTICAL STUDIES OF MODEL PHOTOSYNTHETIC SYSTEMS
}

\author{
Final Progress Report \\ for Period July 1, 1984 to August 31, 1989 \\ University of Nebraska-Lincoln \\ Lincoln, Nebraska 68588-0304
}

Prepared January 15, 1992

Prepared for

THE U.S. DEPARTMENT OF ENERGY

AGREEMENT NO. DE-FG02-84ER13261

\section{NOT I C E}

This report was prepared as an account of work sponsored by the United States Government. Neither the United States nor the Department of Energy, nor any of their employees, nor any of their contractors, subcontractors, or their employees, make any warranty, express or implied, or assumes any legal liability or responsibility for the accuracy, completeness, or usefulness of any information, apparatus, pioduct or process disclosed or represents that its use would not infringe privately-owned rights. 


\begin{abstract}
The objective of this research is to obtain a better understanding of the relationship between the structural organization of photosynthetic pigments and their spectroscopic and electrochemical properties. Defined nodel systems were studied first. These included the least ordered (solutions) through the most highly ordered (Langmuir-Blodgett (LB) monolayers and self-assembled monolayers) systems containing BChl, BPheo, and UQ. Molecules other than the photosynthetic pigments and quinones were also examined, including chromophores (i.e. surface active cyanine dyes and phthalocyanines) and redox active compounds (methyl viologen (MV) and surfactant ferrocenes), in order to develop the techniques needed to study the photosynthetic components. Because the chlorophylls are photosensitive and labile, it was easier first to develop procedures using stable species. Three different techniques were used to characterize these model systems. These included electrochemical techniques for determining the standard oxidation and reduction potentials of the photosynthetic components as well as methods for determining the heterogeneous electron transfer rate constants for $\mathrm{BChl}$ and $\mathrm{BPheo}$ at metal electrodes ( $\mathrm{Pt}$ and $\mathrm{Au}$ ). Resonance Raman (RR) and surface enhanced resonance Raman (SERR) spectroscopy were used to determine the spectra of the photosynthetic pigments and model compounds. SERRS was also used to study several types of photosynthetic preparations.
\end{abstract}

\title{
DISCLAIMER
}

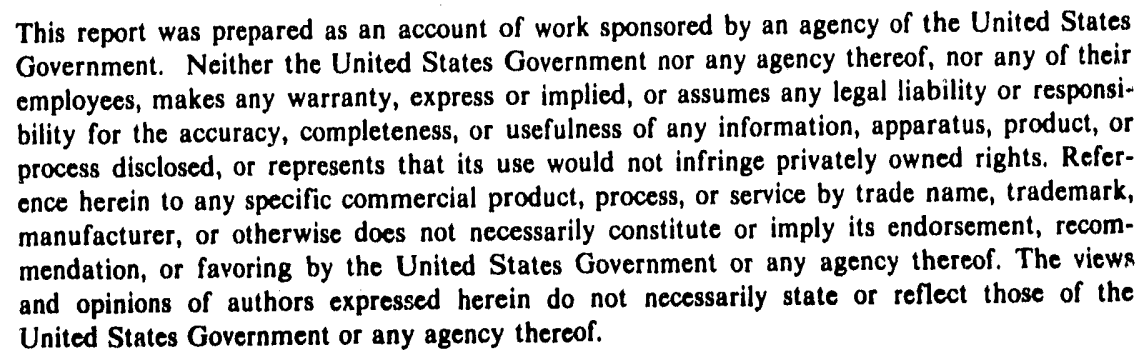




\section{SUMMARY OF RESEARCH PROGRESS}

The results obtained during the above funding period will be summarized within four categories: (1). model systems composed of Langmuir-Blodgett (LB) and self-assembled (SA) monolayers (SA); (2). electrochemical studies; (3) spectroscopic studies; and (4) miscellaneous experiments. The references numbers shown within the summary correspond to the numbers in the Publications section which follows the text.

\section{Model Systems: LB and SA Monolayers $[2,5,12,13,16,20,22,24]$.}

Instrumentation. During the first two years of the funding period, sophisticated monolayer instrumenlation was designed and constructed at the University of NebraskaLincoln. This included a Langmuir trough and film transfer apparatus both of which were interfaced to computer systems. The photolability and chemical instability of BChl monolayers required that these systems be capable of low temperature operation and that the troughs be enclosed and in an inert atmosphere. During the latter part of the funding period covered here, a commercial monolayer trough system was acquired which allowed specialized monolayer manipulations to be undertaken. An optical spectrometer for measuring absorption spectra of a single monolayer was also constructed. This instrument is capable of measuring optical absorption spectra of single monolayers, as well as linear dichroic (LD) spectra. LD spectra are quite essential for determination of orientations of chromophores as well as for orientational studies of helical protein systems such as might be encountered in a chlorophyll-pigment complex.

Surface Isotherms and LB Monolayers. Prior to transfer of spread monolayers to solid supports it is necessary to establish the surface isotherm of the system in order that the film be transferred at the optimum state of compression. Accordingly, isotherms were determined for the compounds to be used in the model systems. These included BChl and BPheo. The two above mentioned compounds were also used at various mole ratios in methyl eicosanate or methyl stearate. Monolayers produced from these multicomponent systems are similar to those to be used in multilayer Langmuir-Blodgett arrays. The data indicate that true molecular miscibility (or true solutions) are present, rather than mosaic patches of pure components. Although this is not an unexpected result, its validity must be established in order to make up more complex systems.

In addition, isotherms were determined for a large number of compounds to be used in the development of electrochemical and optical methods. These included surfactant viologens (with varying hydrocarbon chain lengths) and various combinations of the above compounds. Since phthalocyanines are often taken as good models for porphyrin systems, several of these were also investigated. One of these, with larger hydrocarbon peripheral substitutions than hitherto prepared, gave evidence of a flat, planar orientation in its monolayer (molecular area $\approx 200 \AA^{2}$ ); this appears to be the first phthalocyanine system ever observed which does not assume an edge-on, standing configuration in the monolayer. A second compound, a bifacial, covalently linked dimer was found capable of assuming 
either a flat, closed structure or an extended structure with the macrocycle planes at acute angles to the monolayer plane; configuration was determined by the nature of the spreading solvent used. Aside from the possible value of this compound as a manipulable cofacial bicyclic system with structural features similar to those of the reaction center special pair, the system has novel interest from the viewpoint of fundamental monolayer studies.

The primary thrust of the constructed monolayer systems was based upon BChl, BPheo and other components found in vivo; however, other compounds of potential interest have been prepared. In some cases, these are being used as surrogates or realistic models for the in vivo compounds. To simulate the architecture of the bacterial special pair system, a novel, covalently linked bifacial pheophorbide has been prepared (with $\mathrm{M}$. Wasielewski, Argonne National Laboratory). Additionally, this compound has been made in two forms, one with one long alkyl tail and the other with two such tails. Monolayer results indicate that the two compounds assume different orientations (approximately parallel to and perpendicular to the monolayer plane). Incorporation of these materials into monolaycrs should enable estimates to be made on the effects of orientation on electron transfer. Additional compounds prepared to date include several surface active dyes, useful in studies of energy or electron transfer in monolayer assemblies.

The monolayer behavior of a number of asymmetrically substituted viologens was examined in collaboration with J. Hurst, Oregon Graduate Center. It was found that even those viologens having long alkyl chains (C-12, C-16) were incapable of forming stable monolayers when spread on water subphases (the corresponding bipyridyls did form coherent monolayers). However, stable monolayers were observed with subphases containing concentrated perchlorates (other electrolytes will probably act in a similar manner).

A hydrophobic membrane protein was also found to form stable monolayers at the air/water interface. A protein obtained from eye lens cells, termed MIP 26 (molecular weight is $26 \mathrm{Kdaltons})$. The protein is in the form of a hollow tube $80 \AA$ long, the walls of which are composed of $\alpha$-helices whose exterior surfaces are quite hydrophobic. The interior channel is hydrophilic and rather large (14 $\AA$ diameter); hydrophilic residues are present at both ends of the structure. Such a distribution of hydrophilic and hydrophobic residues allows the protein to orient at the air/water interface. Isotherm data indicates that the structure assumes an end-on orientation on the water surface with the long axis of a cylindrical structure perpendicular to the surface. MIP 26 was also formed into monolayers at electrode surfaces by self assembly along with octadecylmercaptan as matrix material. Preliminary electrochemical experiments were undertaken to demonstrate the action of open and closed channels. Also, a water soluble dye was incorporated into the hydrophilic interior core and the system studied by Raman techniques.

Characterization of the purity' and stability of BChl, BPheo, and the quinones is of primary importance in order that meaningful results be obtained in the monolayer studies. Monomolecular films of these compounds spread on water in a highly attenuated state are 
particularly susceptible to photochemical degradation. Therefore, an HPLC method was developed for monitoring chlorophyll purity during its isolation. Studies of the chemical stability of spread monolayers of BChl under varying the oxidative conditions, time of exposure and substrate $\mathrm{pH}$ were undertaken.

Skeletonized Monolayers or Templates. A new procedure for preparing skeletonized monolayers with specific sites for adsorbing chlorophylls and porphyrins was developed. These templates were prepared by a self-assembly procedure. Electrochemical methods, as well as SERRS, was used to characterize the monolayer template sites. Relationships were established between observed SERRS intensities and density of template sites, as well as the relationship between electrochemically deposited lead ions (underpotential deposition) and the density of template sites.

\section{Electrochemical Studies $[6,9,11,14,14-17,24]$.}

Solution Studies. The electron transfer rate constants for $\mathrm{BChl}$ and $\mathrm{BPheo}$ were determined in organic solvents for the first time. The results showed that the solvent dielectric constant has the greatest effect on the heterogeneous rate constant $\left(\mathrm{k}_{\mathrm{s}}\right)$ for $\mathrm{BChl}$ oxidation at Au or Pt electrodes. The rate constant in methanol was approximately 10 times that in methylene chloride containing sufficient pyridine to disaggregate BChl. The results were interpreted in terms of Marcus theory which relates the solvent dielectric constant to the outer sphere solvent reorganization energy accompanying electron transfer. It was also determined that $\mathrm{BCh}$ self-aggregation or ligand coordination at the central $\mathrm{Mg}$ atom did not have a significant effect on $\mathrm{k}_{\mathrm{s}}$. Using Marcus theory the value of $\mathrm{k}_{\mathrm{s}}$ was used to predict the homogeneous self-exchange rate constant $\left(\mathrm{k}_{\mathrm{h}}\right)$ for $\mathrm{BChl}^{+{ }^{+}}$or $\mathrm{BPheo}^{+}$in solution. The predicted value is orders of magnitude smaller than that observed for various porphyrins. Possible reasons for the difference include the fact that the potential which BChl or BPheo experience in the reaction plane near the electrode surface may be considerably less than that measured by the potentiostat. There is a large drop in potential across the double layer near the electrode surface and the magnitude of this drop depends upon how closely the reactant can approach the electrode. Surface oxides or impurities may also reduce the electron transfer rate.

Adsorbed Species at $\mathrm{Pt}$ and $\mathrm{Ag}$ Electrodes. Adsorption interactions of redox molecules with electrode surfaces can influence electron transfer reactions significantly, as manifest by a shift in the standard redox potential and/or an increase or decrease in the heterogeneous rate constant. A number of quinones (benzoquinone, anthroquinone, and ubiquinone 50) were examined first. Initial studies were concerned with the determination of the redox potentials of the adsorbed quinones using cyclic and differential pulse voltammetry (DPV). Double potential step chronocoulometry (DPSCC) was used to determine the differential surface coverage at two potentials. Large positive shifts in the redox potential of hydroquinone was observed on $\mathrm{Pt}$. The shift was much smaller on $\mathrm{Ag}$. SERS spectra were also obtained for the quinones adsorbed on $\mathrm{Ag}$. 
Photoelectrochemical Studies at $\mathrm{p}$-InP. The photoelectrochemical response of the methylviologen dication $\left(\mathrm{MV}^{2+}\right)$ was studied at $\mathrm{p}$-InP. The objective of these experiments was to characterize the photoclectrochemical properties of the semiconductor using cyclic voltammetry (CV) and differential capacitance measurements. The effect of light intensity or photon flux and photon energy was determined using monochromatic radiation from a $\mathrm{Kr}^{+}$laser. Increasing the photon flux from $26 \mu \mathrm{W}$ to $52 \mathrm{~mW}$ resulted in a positive shift of ca. $600 \mathrm{mV}$ in the cathodic peak maximum. Differential capacitance measurements were used to determine the flatband potential of $\mathrm{p}-\mathrm{InP}$. The differential capacitance results, together with the values of the open-circuit photopotential and band gap, were used to determine the band edges for $\mathrm{p}$-InP. The effect of excitation wavelength on the photopotential was also considered in this study. Three widely different photon energics $(1.65$ to $3.00 \mathrm{eV})$ produced essentially the same photopotentials at the same photon flux. These results suggest that hot electron ejection does not occur under our experimental conditions.

Vesicles. Vesicles composed of surfactants containing asymmetric viologens (methyl group on one nitrogen and $\mathrm{C}_{n} \mathrm{H}_{2 n+1},(n=8,12$, or 16$)$ on the other nitrogen) were also investigated during this period. The electrochemical response of the viologens was characterized by cyclic voltammetry. The objective was to determine the location of the viologen within the vesicle using this approach. According to a previous model based upon homogeneous electron transfer studies, there are two binding sites for the longer chain viologens $\left(\mathrm{C}_{12}\right.$ and $\left.\mathrm{C}_{16}\right)$ in dihexyl phospate vesicles. One site is near the surface of the vesicle and the second is buried within the lipid bilayer. It was determined that the $\mathrm{C}_{16}$ derivative is electroinactive in vesicles, which supports the proposal that it is buried within the bilayer.

\section{Spectroscopic Studies $[1,2,4,5,8,9-11,13,15,18-24]$.}

Solution Raman and Resonance Raman Studies. RR characterization of BChl and BPheo in a range of organic solvents was completed. The purpose was to identify bands which are characteristic of aggregation, ligation, metallation (i.e. $\mathrm{Mg}$ ), and hydrogen bonding interactions. Also, optical and RR studies of BChl and BPheo in detergent solutions were undertaken. Axial coordination sensitive modes were identified in the RR spectra obtained with Soret excitation $(363.8 \mathrm{~nm})$. The major effect of axial ligation on the RR spectrum is the shift of the most prominent band in the spectrum at 1595 to $1609 \mathrm{~cm}^{-1}$. This vibration is within the frequency range previously identified for metalloporphyrin and metallochlorin core-size sensitive vibrations. Other band assignments (e.g the carbonyl modes at $>1630$ $\mathrm{cm}^{-1}$ ) are also sensitive to the solvent.

Characterization of the RR spectra of the radical ions of $B C h l, B P h e o$, and UQ in organic solvents is necessary in order that these species can be identified in vivo. There have been a number of studies of the cation radical of $\mathrm{BChl}$. However, the instability of $\mathrm{BChl}$ and BPheo anion radicals has made RR spectroscopy difficult. The use of a multichannel array detector (OMA II) considerably reduces the time needed to record 
spectra (less than 1 sec) to obtain spectra. Preliminary spectra were obtained of these unstable anion radicals.

Resonance Raman spectroscopy was used to characterize the cation radical of Chl a for the first time. The spectrum is distinct from the neutral species. The carbonyl region of the spectrum was quite similar to the FTIR spectrum of PS II preparations by French researchers. The radical spectrum will be useful for monitoring electron transfer reactions in monolayer assemblies.

Surface Enhanced Raman and Resonance Raman Studies. The goal with respect to SERRS of the photosynthetic pigments is to utilize this technique to monitor electrochemical or photoelectrochemical generation of oxidation and reduction products at an electrode surface. The advantages to SERRS is that it is possible to detect submonolayer amounts of adsorbates at certain metal surfaces. Initial studies of benzoquinone (BQ), UQ and BPheo demonstrated that the SERRS spectrum of the anion radical can be observed.

Photoelectrochemical Studies at $\mathrm{Ag}$ Overlayered $\mathrm{p}$-InP. The potential of SERS/SERRS for monitoring processes at surfaces would be severely constrained if the effect only occurred for adsorbates on the three commonly studied metals. Fortunately, the effect also occurs for surfaces which are overlayered with island films of SERS active metals (Ag has been studied the most). This discovery enables application of SERS to virtually any surface. SERRS could be used to monitor the photoreduction of methyl viologen at p-type InP [22]. This was accomplished by photoelectrochemical or vapor deposition of $\mathrm{Ag}$ islands onto the semiconductor surface. The $\mathrm{Ag}$ islands were characterized by scanning electron microscopy (SEM) and ranged in size from 200 to $400 \AA$. An important conclusion from this study was that the SERR signal tracked the photoelectrochemical response of the InP. Thus, the Ag overlayer did not decrease the photopotential from the value observed for the unmodified electrode (no $\mathrm{Ag}$ overlayer). This observation is crucial to utilizing SERRS to monitor the photoelectrochemical response of the semiconductor.

Langmuir-Blodgett Monolayer Studies. It was demonstrated that SERRS spectra could be obtained of various chromophores (cyanine dyes and chlorophylls) in LB monolayers prepared with fatty acid diluents. Less than 10 mole-percent of the dyes was detected using only $2 \mathrm{~mW}$ of power and spectral integration times less than 1 second. The effect of distance on the intensity of the SERR signal was also investigated using monolayers containing 16 mole-percent of a cyanine dye. Fatty acid spacer layers were used to increase the distance from $27.5 \AA$ (the thickness of 1 fatty acid layer) to $>200 \AA$. The intensity of the dye spectrum decreased exponentially according to electromagnetic theory. The significance of these results is that it should be possible to use SERRS to monitor electron transfer events between redox couples incorporated within adjacent monolayers (e.g. BChl to BPheo).

Vesicle Studies, Vesicles have been used extensively as models for biological membranes. One advantage which vesicles possess over LB monolayers is that it is possible 
to load the interior of the vesicle with electron donors or acceptors and study charge transfer across the hydrocarbon phase using aqueous suspensions. However, the analysis of the results obtained using this approach is often difficult due to the possibility of molecular diffusion across the membrane. The electrochemical and SERRS behavior of heptyl viologen incorporated into various types of vesicles (dihexadecylphosphate (DHP) and phosphatidyl choline (PC)) was studied. It should be possible to detect molecular diffusion using the exquisite surface sensitivity of SERRS. For example, if the inner aqueous phase of the vesicle is loaded with an electron acceptor and the vesicle contains an electron donor at the outer surface, diffusion of either the donor or the acceptor should produce changes in the SERRS intensities accompanying charge transfer. Also, SERRS may provide information about the orientation of the chromophore within the vesicle (perpendicular or parallel to the surface). The enhancement of in-plane or out-of-plane vibrational modes as a function of orientation are predicted from surface selection rules.

IV. Other Results $[3,17]$.

In a preliminary study, SERRS was used to investigate PS II preparations from green plant membranes. The preparations were treated to remove three extrinsic membrane proteins, but not $\mathrm{Mn}$, which is essential to the water splitting activity of these preparations. A low frequency Raman signal was detected under these conditions and it was correlated with the presence of $\mathrm{Mn}$ and $\mathrm{O}_{2}$ evolution activity. The signal may be an indirect monitor of $\mathrm{Mn}$. In a subsequent study, it was postulated that the signal may arise from an $\mathrm{Mn}-\mathrm{O}$ or $\mathrm{Mn}-\mathrm{N}$ vibrational mode. Additional experiments are needed to determine the true origin of this signal.

\section{PUBLICATIONE}

1. Cotton, T. M. "Surface Enhanced Raman Spectroscopy of Biological Macromolecules" in Surface and Interfacial Aspects of Biomedical Polymers, Vol. II, J. D. Andrade, Ed., Plenum Press, 1985 pp. 161-187.

2. Uphaus, R. A., Cotton, T. M., and Möbius, D. "Surface-Enhanced Resonance Raman Spectroscopy of Synthetic Dyes and Photosynthetic Pigments in Monolayer and Multilayer Assemblies" Thin Solid Films 132, 173-184 (1985).

3. Seibert, M. and Cotton, T. M. "A Surface-Enhanced Raman Signal Associated with Manganese in Oxygen-Evolving Photosystem II Membranes" FEBS Lett. 182, 34-38 (1985).

4. Feng, Q. and Cotton, T. M. " A Surface Enhanced Resonance Raman Study of the Photoreduction of Methylviologen on a p-InP Semiconductor Electrode", J. Phys. Chem., 90, 983-987 (1986). 
5. Cotton, T. M., Uphaus, R. A., and Möbius, D. "Distance Dependence of SERS Enhancement in Langmuir-Blodgett Dye Multilayers" J. Phys. Chem. 90, 6071 (1986).

6. Cotton, T. M. and Heald, R. L. "Solvent Effects on the Electron Transfer Kinetics of Bacteriochlorophyll Oxidation" Progress in Photosynthesis Research, J. Biggins, Ed., Martinus Nijhoff Publishers, The Netherlands, 1987, pp. 1.3 333-336.

7. Cotton, T. M. and Heald, R. L. "Solvent Effects on the Heterogeneous Electron Transfer Kinetics of Bacteriochlorophyll $\underline{a}$ and Bacteriopheophytin a at Platinum and Gold Electrodes" J. Phys. Chem. 91 3891-3898 (1987).

8. Callahan, P. M. and Cotton, T. M. "Assignment of Bacteriochlorophyll a Ligation State from Absorption and Resonance Raman Spectra" J. Am. Chem. Soc. 109, 70017007 (1987).

9. Lu, T. and Cotton, T. M. "In situ Raman Spectra of the Three Redox Forms of Heptylviologen at Pt and Ag Electrodes: Counterion Effects" J. Phys. Chem. 21, 5978-5985 (1987).

10. Cotton, T. M. "The Application of Surface Enhanced Raman Scattering to Biochemical Systems" Spectroscopy of Surfaces, (Advances in Spectroscopy, Vol. 15, R. J. H. Clark and R. E. Hester, Eds.) Wiley, 1988, p. 91-153.

11. Feng, Q. and Cotton, T. M. "A Study of Charge Transfer at an Illuminated p-InP Electrode in Aqueous Solution" J. Elecirochem. Soc. 135, 591-598 (1988).

12. Kim, J.-H., Cotton, T. M., and Uphaus, R. A. "Monolayer Characteristics of Some Multinucleate Phthalocyanines" Thin Solid Films, 159, 141-147 (1988).

13. Kim, J.-H.,Cotton, T. M., and Uphaus, R. A. "Molecular Recognition in Monolayers and Species Detection by Surface Enhanced Resonance Raman Spectroscopy" Thin Solid Films, 160, 389-397 (1988).

14. Lu, T. and Cotton, T. M. "Voltammetric Study of Asymmetric Viologens in an Organic Solvent, Aqueous Solution, and Vesicle Systems" J. Electroanal. Chem., 246, 337-347 (1988).

15. Heald, R. H., Callahan, P. M., and Cotton, T. M. "Resonance Raman Spectra of Electrochemically Generated Chlorophyll a, Cation Radical" J. Phys. Chem. 92, 48204824 (1988).

16. Kim, J.-H., Cotton, T. M., and Uphaus, R. A. "Electrochemical and Raman Characterization of Molecular Recognition Sites in Self-Assembled Monolayers" J. Phys. Chem. 22, 5575-5578 (1988). 
17. Seibert, M., Cotton, T. M., and Metz, J. G. "Surface-Enhanced Raman Scattering Spectroscopy: Probing the Lumenal Surface of Photosystem II Membranes for Evidence of Manganese" Biochim. Biophys. Acta 934, 235-246 (1988).

18. Lu, T., Cotton, T. M., Hurst, J. K., and Thompson, D. H."P. "A Raman and SurfaceEnhanced Raman Study of Asymmetrically-Substituted Viologens" J. Phys. Chem. 22, 6978-6985 (1988).

19. Lu, T., Cotton, T. M., Birke, R. L., and Lombardi, J. R. "Raman and SurfaceEnhanced Raman Spectruscopy of the Three Redox Forms of 4,4'-Bipyridine" Langmuir 5, 406-414 (1989). Correction: Langmuir 5, 886 (1989).

20. Kim, J.-H., Cotton, T. M., Uphaus, R. A., and Möbius, D. "Surface-Enhanced Resonance Raman Scattering from Langmuir-Blodgett Monolayers: Surface Coverage-Intensity Relationships" J. Phys. Chem. 93, 3713-3720 (1989).

21. Feng, Q., Yue, W. and Cotton, T. M. "SERRS of Methylviologen at Silver Electrodes" J. Phys. Chem. 94, 2082-2091 (1990).

22. Kim, J.-H., Cotton, T. M., and Uphaus, R. A. "Monolayer Molecular Recognition Sites as a Basis for Biosensor Development" in Proceedings of the Symposium on Molecular Electronics, Santa Clara, CA, 1988, F. T. Hong, Ed., Plenum Press, New York.

23. Heald, R. L. and Cotton, T. M. "A Resonance Raman Investigation of the Cation Radical of Chlorophyll a and Several Derivatives" J. Phys. Chem., 24, 3986-3975 (1990).

24. Cotton, T. M., Kim, J.-H., and Uphaus, R. A. "Spectroscopic and Electrochemical Studies of Oriented Monolayers on Electrode Surfaces" Microchem. J., 42, $44-71$ (1990). 

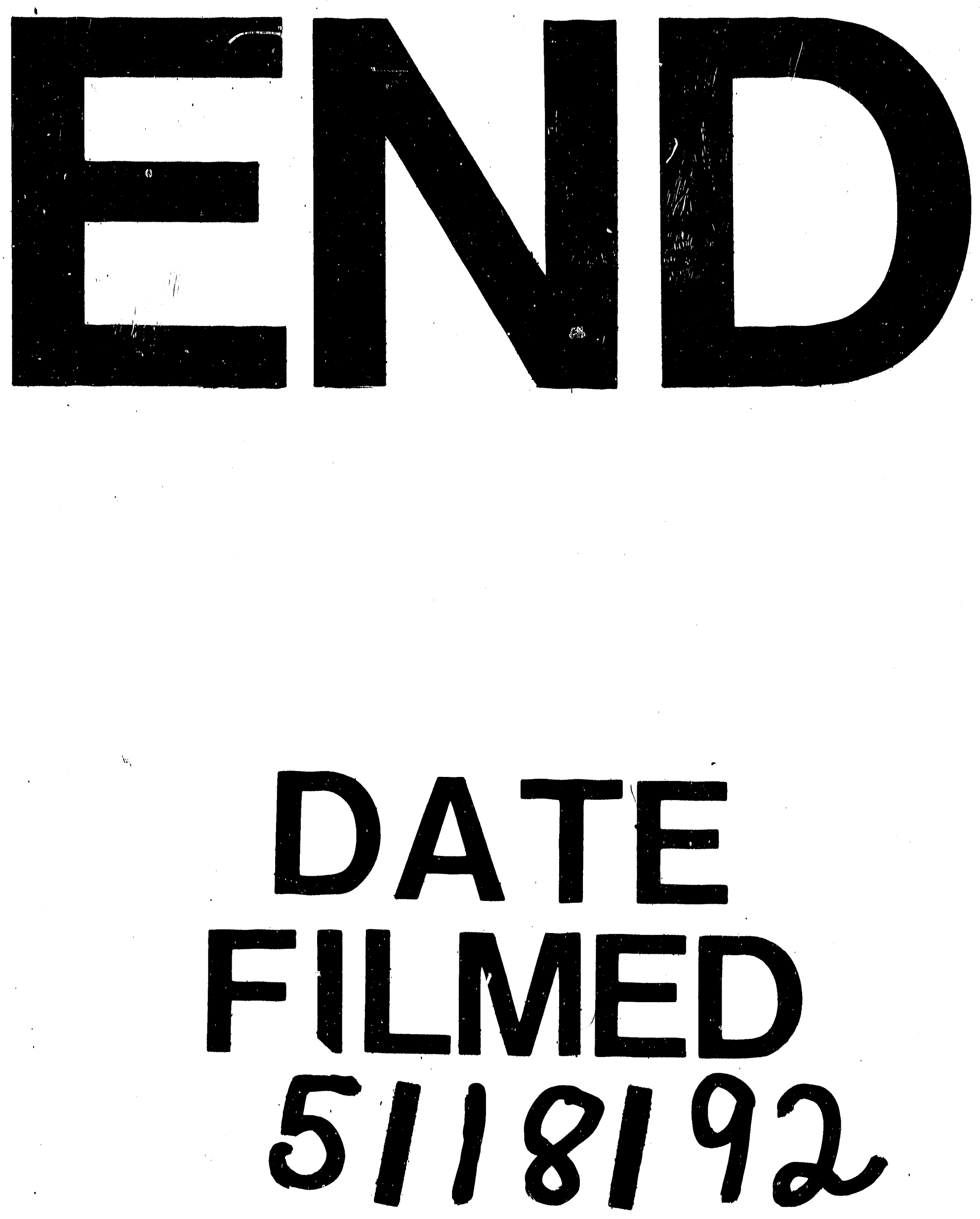
